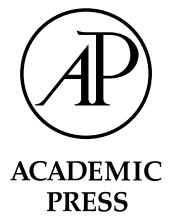

\title{
Regulation of Toll-like receptor 4 signalling by A20 zinc finger protein
}

\author{
Susan M. O'Reilly and Paul N. Moynagh* \\ Department of Pharmacology, Conway Institute of Biomolecular and Biomedical Research, University College Dublin, Belfield, Dublin 4, Ireland
}

Received 10 February 2003

\begin{abstract}
A20 is a zinc finger protein that renders cells resistant to apoptosis. However, the recent demonstration that A20-deficient mice develop severe inflammation and are hyper-responsive to LPS suggests that A20 may play a key role in regulating the inflammatory response. This study, for the first time, explores the likely mechanism by which A20 can regulate the pro-inflammatory effects of LPS. More specifically it characterises the ability of A20 to modulate TLR-4 signalling since TLR-4 acts as the signalling receptor system for LPS. Full length A20 inhibited the ability of TLR-4 to activate the transcription factors, NF- $\mathrm{kB}$ and AP-1, and induce the chemokine IL-8. The inhibitory capacity of A20 on NF- $\mathrm{kB}$ was localised to the C-terminal zinc finger domain of A20 whereas full length A20 was required to effect inhibition of AP-1 and IL-8. Furthermore full length and C-terminal A20 showed similar regulatory effects on MEKK-1 activation of NF- $\mathrm{KB}$ and AP-1 and induction of IL-8. The findings increase our mechanistic understanding of the anti-inflammatory effects of A20 and suggest that it modulates TLR-4 signalling at or downstream of MEKK-1. (C) 2003 Elsevier Science (USA). All rights reserved.
\end{abstract}

Keywords: Inflammation; Signal transduction; Transcription factors; Toll-like receptor

A20 is a novel $90 \mathrm{kDa}$ zinc finger protein that is induced by a range of stimuli including IL-1, TNF, CD40, and LMP-1 [1-3]. The induction of A20 by these stimuli tends to render cells resistant to apoptosis $[2,3]$. The transcription factor $\mathrm{NF}-\mathrm{\kappa B}$ is activated by all of these agents and plays a key role in regulating the expression of A20 [1-4]. Interestingly A20 in turn acts to inhibit activation of NF- $\kappa \mathrm{B}$ and this serves as an auto-regulatory negative feedback system [5]. Since NF- $\kappa B$ regulates the expression of a plethora of pro-inflammatory genes much interest has focussed on the mechanisms by which A20 inhibits its activation by IL-1 and TNF. The C-terminal region of A20 contains seven Cys $2 / \mathrm{Cys} 2$ zinc fingers and this region is responsible for effecting inhibition of NF-кB [6-8]. Whilst the mechanism underlying such inhibitory activity is incompletely understood, the $\mathrm{C}$-terminal zinc finger domain is known to associate with A20-binding inhibitor of NF-KB (ABIN)-1 and -2 $[9,10]$. Both ABIN proteins can mimic the inhibitory effects of $A 20$ on NF- $\mathrm{B}$ suggesting that they might mediate the inhibitory effects of A20. Furthermore the

\footnotetext{
${ }^{*}$ Corresponding author. Fax: +353-1-269-2749.

E-mail address: P.Moynagh@ucd.ie (P.N. Moynagh).
}

C-terminal region of A20 also interacts with NEMO $(\mathrm{I} \kappa \mathrm{B}$ kinase $(\mathrm{IKK}) \gamma$ ) [11]. Since the latter is a key scaffolding protein in the IKK complex, that promotes activation of $\mathrm{NF}-\mathrm{\kappa B}$, the interaction of the C-terminal domain of A20 with NEMO may represent another important association in mediating the inhibitory effects of A20 on NF- $\mathrm{BB}$. The N-terminal region of A20 also interacts with other signalling components in the NF- $\mathrm{KB}$ pathway. Thus A20, through its $\mathrm{N}$-terminal domain, can directly interact with TNF-receptor-associated factors (TRAF)-1, TRAF-2 [8], and TRAF-6 [7]. However the latter report demonstrated that a truncated form of A20, lacking the N-terminal TRAF-interacting domain, retained the ability to inhibit IL-1 and TNF activation of $\mathrm{NF}-\kappa \mathrm{B}$. This suggests that TRAF binding is not necessary for mediating the inhibitory effects of A20 on $\mathrm{NF}-\kappa \mathrm{B}$.

A20 is also known to inhibit the activation of the transcription factor AP-1 [5]. However the regions of A20 that mediate such inhibition have yet to be delineated. The dualist role of A20 in inhibiting both NF- $\mathrm{KB}$ and AP-1 suggests that it may target a signalling component that promotes activation of both transcription factors. One such molecule may be MEKK-1 since it is 
known to mediate activation of both NF- $\mathrm{KB}$ and AP-1 $[12-14]$ and a recent report has suggested that A20 inhibits MEKK-1 activation of NF- $\mathrm{BB}$ [15]. However the ability of A20 to inhibit MEKK-1 activation of AP-1 was not addressed.

The induction of A20 confers on NF- $\mathrm{BB}$ the ability to regulate its own activation and since NF- $\kappa \mathrm{B}$ is a pivotal player in the generation of the inflammatory response, A20 is likely to be an endogenous regulator of inflammation. This has recently been confirmed by a study showing that A20-deficient mice develop severe inflammation due to persistent activation of $N F-\kappa B$ [16]. Furthermore these mice are hyper-responsive to LPS suggesting that A20 has the capacity to regulate the inflammatory response to gram-negative bacteria. However the mechanism by which A20 modulates LPS responsiveness was not addressed. Toll-like receptor 4 (TLR-4) acts as the receptor for LPS in gram-negative bacteria and in doing so effects the induction of proinflammatory proteins [17-19]. TLR-4 employs many of the same signalling components as the IL-1 receptor system and like IL-1 can activate NF- $\kappa$ B and AP-1 [20]. Since A20 is known to regulate the activation of these transcription factors by IL-1 we sought to examine the ability of A20 to inhibit TLR-4 activation of NF- $\kappa B$ and AP-1 with a view to increasing our understanding of the anti-inflammatory effects of A20. We also aimed to identify the regions of A20 that are responsible for mediating its anti-inflammatory effects. We show that A20 inhibits the ability of TLR-4 to activate NF- $\kappa$ B and AP-1 and that the C-terminal zinc finger domain of A20 retains the ability to inhibit TLR-4 activation of $\mathrm{NF}-\kappa \mathrm{B}$. However the C-terminal domain is ineffective in regulating activation of AP-1 by TLR-4 and fails to mimic the inhibitory effects of full length A20 on TLR4 induction of IL-8. Finally we present data in which A20 may target MEKK-1 in the TLR-4 signalling pathway.

\section{Materials and methods}

Materials. HEK293 cells were obtained from European Collection of Animal Cell Cultures (Salisbury, UK). DMEM, penicillin, streptomycin, trypsin, and foetal calf serum were from Gibco (Grand Island, NY, USA). The Reporter Lysis Buffer, Luciferase Assay System, and Pfu were from Promega Corporation (WI, USA). EcoRI, XbaI, and T4 ligase were from New England Biolabs (Hertfordshire, UK). TOP10 chemically competent Escherichia coli, the plasmid encoding the dominant positive mutant of MEKK-1 (pFC-MEKK-1), and the AP-1-luciferase plasmid (AP-1-luc) were obtained from Stratagene (La

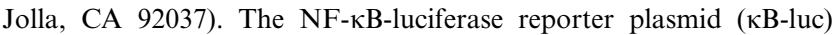
consists of five copies of the NF- $\mathrm{KB}$ consensus site cloned into the luciferase reporter construct pGL3-Basic (Promega). pcDNA3.1 was obtained from Invitrogen (Groningen, The Netherlands). P3xFLAGMyc-CMV was from Sigma (Poole, UK). Polyfect transfection agent was from Qiagen (West Sussex, UK). Matched antibody pairs against IL-8 and recombinant human IL-8 (standard) were from R\&D
Systems Europe (Abingdon, Oxford, UK). CD4-TLR4 cDNA, A20pJDM, and the rabbit anti-human A20 antibody were gifts from Dr. Charles A. Janeway, Jr. (Yale University, USA), Dr. Dermot Walls (Dublin City University, Ireland), and V.M. Dixit (Genentech, USA), respectively.

Cell culture. HEK293 cells were cultured in DMEM containing $10 \%(\mathrm{v} / \mathrm{v})$ foetal calf serum, glutamine $(2 \mathrm{mM})$, penicillin $(100 \mathrm{U} / \mathrm{ml})$, and streptomycin $(100 \mu \mathrm{g} / \mathrm{ml})$. The cells were incubated at $37^{\circ} \mathrm{C}$ in an atmosphere of $5 \% \mathrm{CO}_{2}$. Cells were passaged using $0.25 \%(\mathrm{w} / \mathrm{v})$ trypsin in DMEM.

Cloning of C-terminal domain of A20. The $1251 \mathrm{bp}$ fragment of the gene encoding all seven zinc finger domains of the C-terminal domain of A20 was amplified from the A20pJDM template using Pfu polymerase in a polymerase chain reaction. The forward and reverse primers contained $E c o$ RI and $X b a \mathrm{I}$ sites, respectively. The sequence of the primers were:

Forward primer: $5^{\prime}$-CAT GGA ATT CAG CTT CCG TGC CCC AGC TT- $3^{\prime}$

Reverse primer: $5^{\prime}$-CTA GTC TAG CAT ACA TCT GCT TGA ACT GAA A- $3^{\prime}$

The reaction was initially heated to $94^{\circ} \mathrm{C}$ for $3 \mathrm{~min}$ and then subjected to 25 cycles of the following sequential steps: $94^{\circ} \mathrm{C}$ for $1 \mathrm{~min}$, $65^{\circ} \mathrm{C}$ for $1 \mathrm{~min}$, and $72^{\circ} \mathrm{C}$ for $5 \mathrm{~min}$. The products were subjected to agarose electrophoresis and the $1251 \mathrm{bp}$ fragment excised and purified using glass wool. The purified PCR fragment and the p3XFLAGMyc-CMV expression vector were both digested with EcoRI (10 U) and $X b a \mathrm{I}(10 \mathrm{U})$ to produce the cohesive ends necessary for ligation. The reaction mixtures were heated to $65^{\circ} \mathrm{C}$ for $20 \mathrm{~min}$ to inactivate both enzymes. The digested C-terminal A20 fragment was precipitated from solution and the digested vector was purified using the Qiagen gel extraction kit according to the manufacturer's instructions. A ligation reaction was set up using a 5:1 ratio of insert to vector and $\mathrm{T}_{4}$ ligase enzyme $(3 \mathrm{U})$. The reaction was incubated overnight at $4{ }^{\circ} \mathrm{C}$ and the ligation mix was then used to transform TOP10 chemically competent $E$. coli. Plasmid DNA was generated and the presence of the C-terminal A20 insert was confirmed by PCR using conditions as described above. Plasmid DNA containing the insert was then subjected to commercial sequence analysis at GATC $\mathrm{GmbH}$ sequencing laboratories.

Transfection of HEK293 cells. HEK293 cells were seeded at $3 \times 10^{5}$ cells $/ \mathrm{ml}(3 \mathrm{ml})$ in complete DMEM in 6-well tissue culture dishes and grown for $24 \mathrm{~h}$. Transfections were performed with the designated amounts of the appropriate plasmids using Polyfect transfection reagent (Qiagen) according to the manufacturer's recommendations. The plasmids included: NF- $\mathrm{BB}$-regulated luciferase reporter $(0.5 \mu \mathrm{g})$, AP-1 regulated luciferase reporter $(0.5 \mu \mathrm{g})$, CD4/TLR4 $(1 \mu \mathrm{g})$, MEKK-1 (50-200 ng), A20pJDM $(1 \mu \mathrm{g})$, and C-terminal A20p3xFLAG-Myc-CMV $(1 \mu \mathrm{g})$. The total amount of DNA in each transfection was $3.6 \mu \mathrm{g}$ and pcDNA3.1(+) was used to supplement the total DNA to this level. Cells were allowed to recover for $24 \mathrm{~h}$ after the transfection and the medium was removed and replaced with complete DMEM. The cells were then allowed to grow for another $6 \mathrm{~h}$. Conditioned medium was retained for IL-8 measurement (see below). Cell extracts were generated and measured for luciferase activity using the Luciferase Assay System with Reporter Lysis Buffer from Promega Corporation. Extracts were also assayed for protein content [21] and these values were used for normalising luciferase readings.

Western immunoblotting of transfected cells. HEK293 cells were electroporated as above with A20pJDM or C-terminal A20p3xFLAG-Myc-CMV. The cells were then grown for $24 \mathrm{~h}$. The 6-well dishes were then placed on ice, the medium removed, and the cells washed twice with ice-cold PBS $(1 \mathrm{ml})$. The cells were then incubated for $30 \mathrm{~min}$ in ice-cold lysis buffer $(250 \mu \mathrm{l})(20 \mathrm{mM}$ Tris-HCl buffer, $\mathrm{pH}$ 7.5 , containing $150 \mathrm{mM} \mathrm{NaCl}, 1 \%$ (v/v) Triton, $1 \mathrm{mM}$ EDTA, $1 \mathrm{mM}$ $\mathrm{Na}_{3} \mathrm{VO}_{4}, 1 \mathrm{mM}$ DTT, $1 \mathrm{mM}$ PMSF, and $1 \mathrm{mM}$ of protease inhibitor mix of leupeptin, aprotonin, benzamidine, and trypsin inhibitor). Cells 
were scraped and pelleted by centrifugation at $12,000 \mathrm{~g}$ for $10 \mathrm{~min}$ at $4{ }^{\circ} \mathrm{C}$. The resulting supernatants were retained and their protein content was measured as above. Samples $(30 \mu \mathrm{g}$ protein) were then mixed with SDS-PAGE sample buffer $(50 \mathrm{mM}$ Tris- $\mathrm{HCl}$ buffer, $\mathrm{pH} 6.8$ containing 2\%(w/v) SDS, $10 \%(\mathrm{w} / \mathrm{v})$ glycerol, $1 \%(\mathrm{v} / \mathrm{v}) 2$-mercaptoethanol, and $0.1 \%(\mathrm{w} / \mathrm{v})$ bromophenol blue) and heated to $90{ }^{\circ} \mathrm{C}$ for $5 \mathrm{~min}$. Lysates were cleared of particulate material by centrifugation at $20,000 \mathrm{~g}$ for $5 \mathrm{~min}$. The clear supernatants were subjected to SDSPAGE on $10 \%$ polyacrylamide slab gels and the separated proteins electrophoretically transferred from the gels to nitrocellulose. The presence of full length and C-terminal A20 expression was detected by probing the nitrocellulose with a rabbit anti-human A20 polyclonal antibody (1/500 dilution) and visualising using a horseradish peroxidase-conjugated donkey anti-rabbit antibody in conjunction with an enhanced chemiluminescence system (Pierce) according to the manufacturer's instructions.

Assays of $I L-8$. Conditioned medium from cells (see above) was assayed for IL-8 using a standard "sandwich type" ELISA. The ELISA assay utilised matched antibody pairs and recombinant human IL-8 as standard and was performed according to the manufacturer's instructions (R\&D Systems).

Statistical analysis. Significance was evaluated by Student's $t$ test for paired data.

\section{Results}

Full length A20 inhibits TLR-4 activation of $N F-\kappa B$ and $A P-1$ and induction of $I L-8$

The hyper-responsive nature of A20-deficient mice to LPS coupled to their susceptibility to develop severe inflammation emphasises the anti-inflammatory potential of A20. In order to explore the molecular mechanism underlying the regulatory effects of A20 on LPS and inflammation, the present study initially examined the ability of A20 to modulate the activation of the transcription factors, NF- $\mathrm{BB}$ and AP-1, by TLR-4. HEK293 cells were thus co-transfected with CD4/TLR4, the constitutively active form of TLR4 [17], in the absence and presence of A20pJDM, a mammalian expression construct that constitutively expresses full length A20. The overexpression of A20 was confirmed by the presence of a $90 \mathrm{kDa}$ band that was reactive with a rabbit anti-human A20 polyclonal antibody in western immunoblotting analysis of extracts from transfected cell populations (Fig. 1A). The lack of detectable A20 in HEK293 cells that had not been transfected with A20pJDM indicates low or absent basal expression of A20 in these cells. The overexpressed A20 was initially examined for its ability to regulate CD4/TLR4 activation of NF- $\kappa$ B (Fig. 1B). The activity of the latter was determined by measuring the induction of a transfected $\mathrm{NF}-\kappa \mathrm{B}-$-regulated luciferase gene ( $\kappa \mathrm{B}-\mathrm{luc})$. The luciferase expression was induced 6-fold when cells were cotransfected with CD4/TLR4. However, the overexpression of full length A20 was sufficient to abolish the CD4/ TLR4 induction of luciferase. A20 was next assessed for its capacity to modulate activation of AP-1 by TLR-4
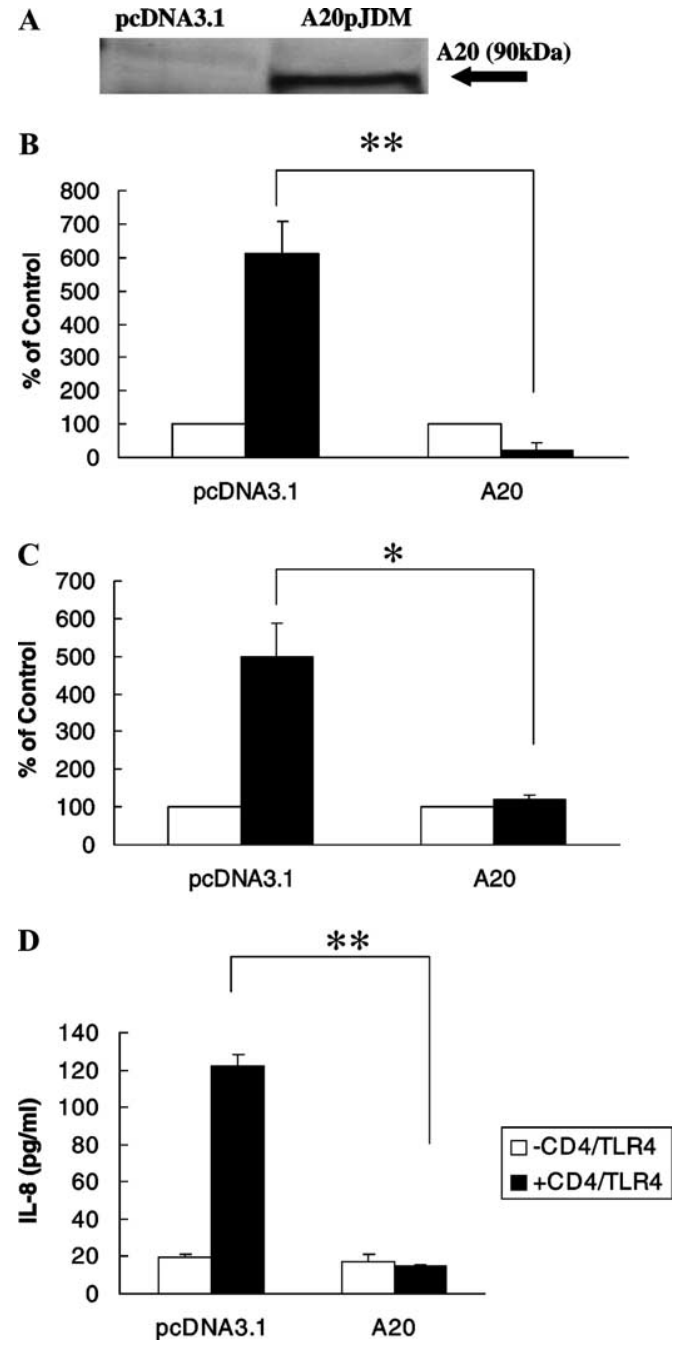

Fig. 1. Effects of full length A20 on TLR-4 signalling. (A) HEK293 cells were transfected with pcDNA3.1(+) or A20pJDM $(1 \mu \mathrm{g})$. Cell lysates were subsequently subjected to SDS-PAGE on a $10 \%$ polyacrylamide gel and electroblotted onto nitrocellulose membrane. The presence of full length A20 expression was detected by probing the nitrocellulose with a rabbit anti-human A20 polyclonal antibody (1/ 500 dilution) and visualising by enhanced chemiluminescence. (B,C) HEK293 cells were co-transfected with or without CD4/TLR4 $(1 \mu \mathrm{g})$ in the presence or absence of A20pJDM $(1 \mu \mathrm{g})$ and $(\mathrm{B}) \kappa \mathrm{B}-\mathrm{luc}(0.5 \mu \mathrm{g})$ or (C) AP-1-luc $(0.5 \mu \mathrm{g})$. Cell extracts were subsequently measured for luciferase activity and protein content. Data are presented relative to cells transfected in the absence of CD4/TLR4 (D) HEK293 cells were co-transfected with or without CD4/TLR4 $(1 \mu \mathrm{g})$ in the presence or absence of A20pJDM $(1 \mu \mathrm{g})$. Conditioned medium from cells was then assayed for IL- 8 by ELISA. Results represent means \pm SEM of three independent experiments $(*, p<0.05 ; * *, p<0.01)$.

(Fig. 1C). The activity of AP-1 was determined by measuring the expression of a transfected AP-1 luciferase construct (AP-1-luc). The cells that were transfected with CD4/TLR-4 showed a 5-fold induction of AP-1 regulated luciferase relative to a basal expression. However, like NF- $\mathrm{BB}$, the expression of the AP-1-regulated luciferase by CD4/TLR4 was completely abro- 
gated when cells were co-transfected with A20pJDM. Thus A20 is a very effective inhibitor of TLR-4 activation of NF- $\kappa \mathrm{B}$ and $\mathrm{AP}-1$. In order to appreciate the functional relevance of the inhibitory effects on A20 on TLR-4 activation of NF- $\mathrm{KB}$ and AP-1 we examined the effects of A20 on the ability of TLR-4 to induce IL-8 (Fig. 1D). The transfection of HEK293 cells with CD4/ TLR4 was sufficient to effect a 6-fold induction of IL-8. However, the overexpression of full length A20 abolished this induction again emphasising the anti-inflammatory effects of A20.

\section{$C$-terminal zinc finger domain of A20 inhibits TLR-4 activation of $N F-\kappa B$ but not $A P-1$}

We then sought to identify the regions of A20 that mediate the above regulatory effects on TLR-4 signalling. It was already known that the C-terminal zinc finger domain of A20 is sufficient to inhibit IL-1 activation of $\mathrm{NF}-\kappa \mathrm{B}$. The present study thus explored the ability of C-terminal A20 to mimic full length A20 in its abolition of TLR-4 activation of NF-kB. Furthermore there was no existing data on the ability of C-terminal domain to regulate AP-1 activation by any stimuli and thus this novel aspect was also addressed in the present project. The region of the A20 gene encoding all seven zinc finger domains of the C-terminal domain of A20 was thus amplified by PCR and subcloned into the mammalian expression vector p3xFLAG-Myc-CMV. The resulting construct was used to overexpress the C-terminal region of A20 in HEK293 cells. The overexpression of the truncated A20 was evident by the presence of a $48 \mathrm{kDa}$ band that reacted with the same rabbit anti-human A20 polyclonal antibody as used above (Fig. 2A). The expression vector encoding the $\mathrm{C}$-terminal domain of A20 was thus used to probe the ability of this region of A20 to regulate TLR-4 activation of $N F-\kappa B$ and AP-1. The overexpression of C-terminal A20 caused significant inhibition of TLR-4 activation of NF-кB (Fig. 2B). However, unlike full length A20 the inhibition was incomplete. Furthermore the $\mathrm{C}$-terminal region of A20 failed to mimic full length A20 in that it failed to show any modulatory effects on TLR-4 activation of AP-1 (Fig. 2C). Thus whilst the C-terminal region of A20 is sufficient to inhibit TLR-4 activation of NF- $\kappa B$, full length A20 is required to manifest inhibition of TLR-4 activation of AP-1. The C-terminal zinc finger domain of A20 was also assessed for its capacity to modulate TLR-4 induction of IL- 8 in order to assess the consequence of its inability to regulate AP-1 on its anti-inflammatory potential (Fig. 2D). The C-terminal domain was ineffective in modulating IL-8 induction by TLR- 4 suggesting that the ability of full length A20 to inhibit AP-1 is a key component in mediating its anti-inflammatory activity.
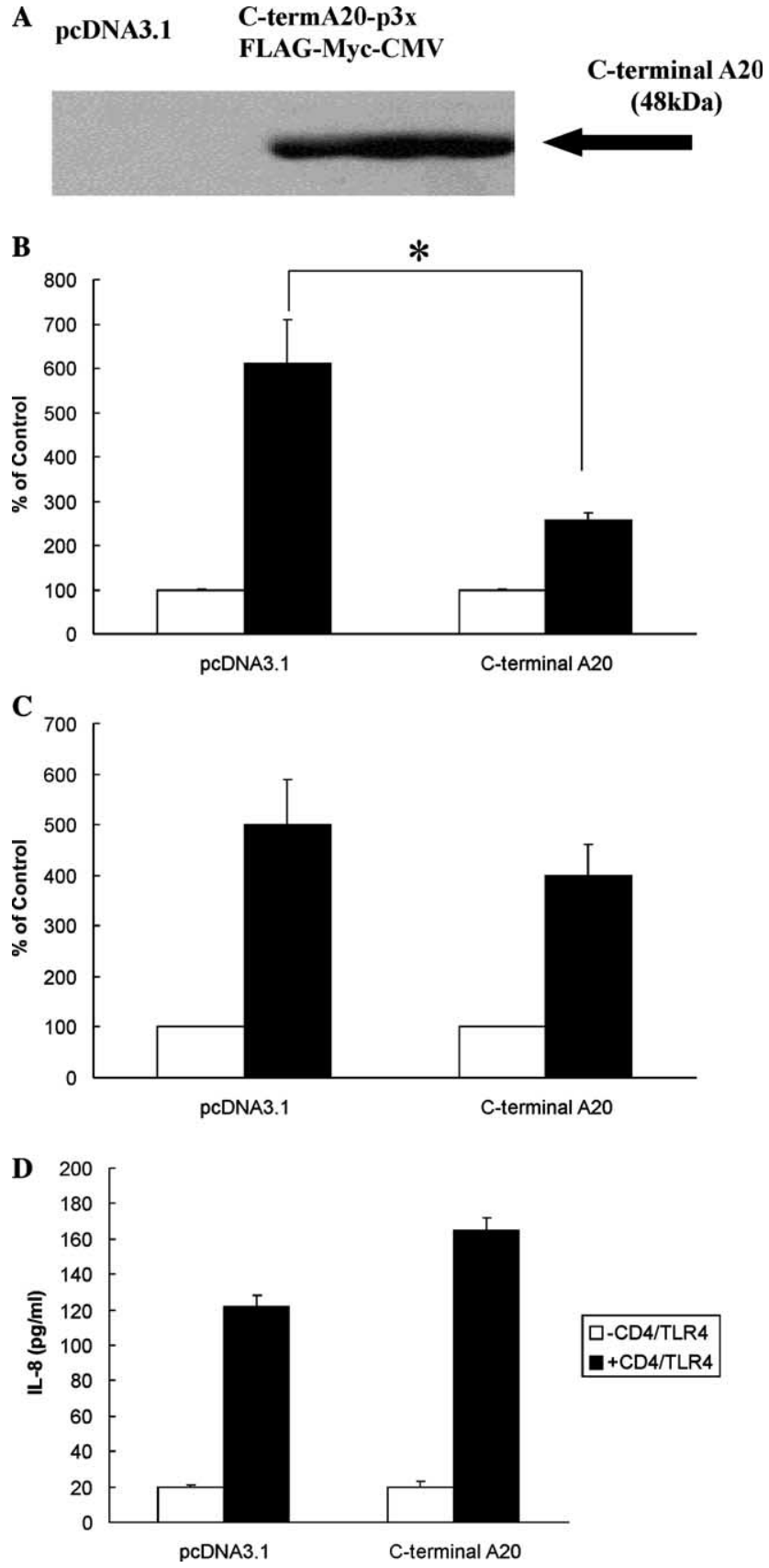

Fig. 2. Effects of C-terminal A20 on TLR-4 signalling. (A) HEK293 cells were transfected with pcDNA3.1(+) or C-terminal A20p3xFLAG-Myc-CMV $(1 \mu \mathrm{g})$. Cell lysates were subsequently subjected to SDS-PAGE on a $10 \%$ polyacrylamide gel and electroblotted onto nitrocellulose membrane. The presence of C-terminal A20 expression was detected by probing the nitrocellulose with a rabbit anti-human A20 polyclonal antibody (1/500 dilution) and visualising by enhanced chemiluminescence. (B,C) HEK293 cells were co-transfected with or without CD4/TLR4 $(1 \mu \mathrm{g})$ in the presence or absence of C-terminal A20-p3xFLAG-Myc-CMV $(1 \mu \mathrm{g})$ and (B) $\kappa \mathrm{B}-\mathrm{luc}(0.5 \mu \mathrm{g})$ or (C) AP-1luc $(0.5 \mu \mathrm{g})$. Cell extracts were subsequently measured for luciferase activity and protein content. Data are presented relative to cells transfected in the absence of CD4/TLR4. (D) HEK293 cells were cotransfected with or without CD4/TLR4 $(1 \mu \mathrm{g})$ in the presence or absence of C-terminal A20-p3xFLAG-Myc-CMV $(1 \mu \mathrm{g})$. Conditioned medium from cells was then assayed for IL- 8 by ELISA. Results represent means \pm SEM of three independent experiments $\left({ }^{*}, p<0.05\right)$. 
Full length A20 inhibits MEKK-1 activation of $N F-\kappa B$ and $A P-1$ and induction of $I L-8$

We next attempted to identify a target for A20 in mediating its inhibitory effects on TLR-4. MEKK-1 was selected as a lead candidate since it is known to regulate activation of both NF- $\mathrm{KB}$ and AP-1. Full length A20 was thus assessed for its ability to regulate MEKK-1 activation of NF- $\kappa$ B and AP-1 and induction of IL-8 in HEK293 cells (Fig. 3). A plasmid encoding a dominant positive form of MEKK-1, when co-transfected with the КB-luc and AP-1-luc constructs, caused a 6-fold and 140 -fold induction of luciferase, respectively. The overexpression of full length A20 caused a total abolition of the induction of $\kappa \mathrm{B}$-luc (Fig. 3A) and a partial inhibition of AP-1-luc (Fig. 3B). The regulatory effects of full length A20 on MEKK-1 induction of IL-8 was also
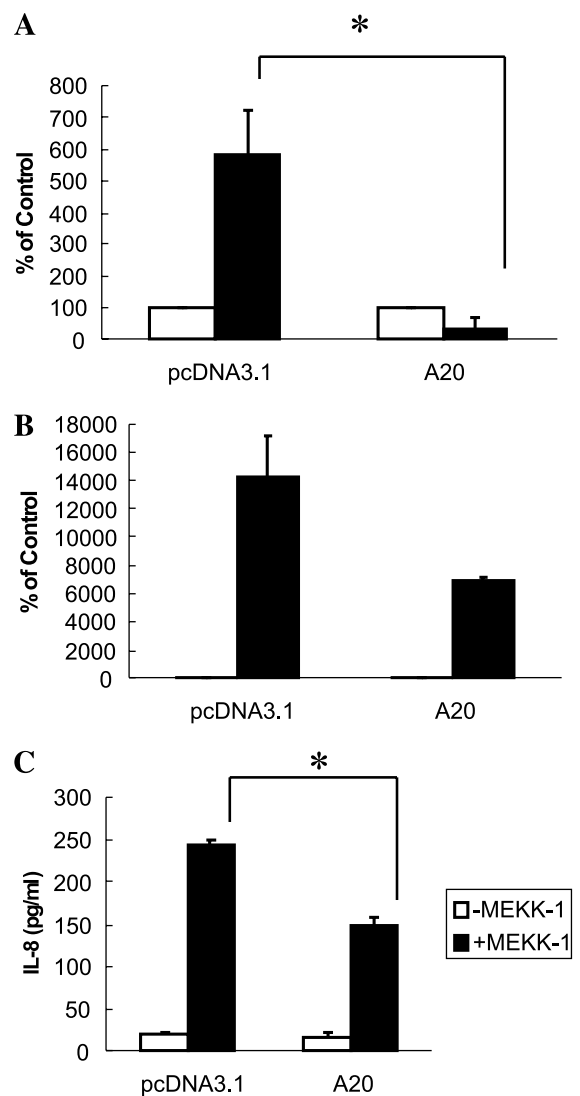

Fig. 3. Effects of full length A20 on MEKK-1 signalling. (A,B) HEK293 cells were co-transfected with or without MEKK-1 (200 ng in (A); $50 \mathrm{ng}$ in (B)) in the presence or absence of A20pJDM $(1 \mu \mathrm{g})$ and (A) $\kappa \mathrm{B}-\mathrm{luc}(0.5 \mu \mathrm{g})$ or (B) AP-1-luc $(0.5 \mu \mathrm{g})$. Cell extracts were subsequently measured for luciferase activity and protein content. Data are presented relative to cells transfected in the absence of MEKK-1. (C) HEK293 cells were co-transfected with or without MEKK-1 (200 ng) in the presence or absence of A20pJDM $(1 \mu \mathrm{g})$. Conditioned medium from cells was then assayed for IL-8 by ELISA. Results represent means $\pm \mathrm{SEM}$ of three independent experiments $(*, p<0.05)$. assessed (Fig. 3C). The transfection of HEK293 cells with MEKK-1 was sufficient to effect a 12 -fold induction of IL-8. However the overexpression of full length A20 reduced this induction. Thus the inhibitory effects of A20 on MEKK-1 activation of NF- $\kappa$ B and AP- 1 and induction of IL-8 mimicked its effects on TLR-4 suggesting that A20 inhibits TLR-4 at or downstream of MEKK-1.

$C$-terminal zinc finger domain of A20 inhibits MEKK-1 activation of $N F-\kappa B$ but not AP-1

In a scenario where A20 inhibits TLR-4 at or downstream of MEKK-1, one would predict that the C-terminal region of A20 should also regulate MEKK1 in a manner similar to its modulation of TLR-4. This was thus explored by examining the effects of the
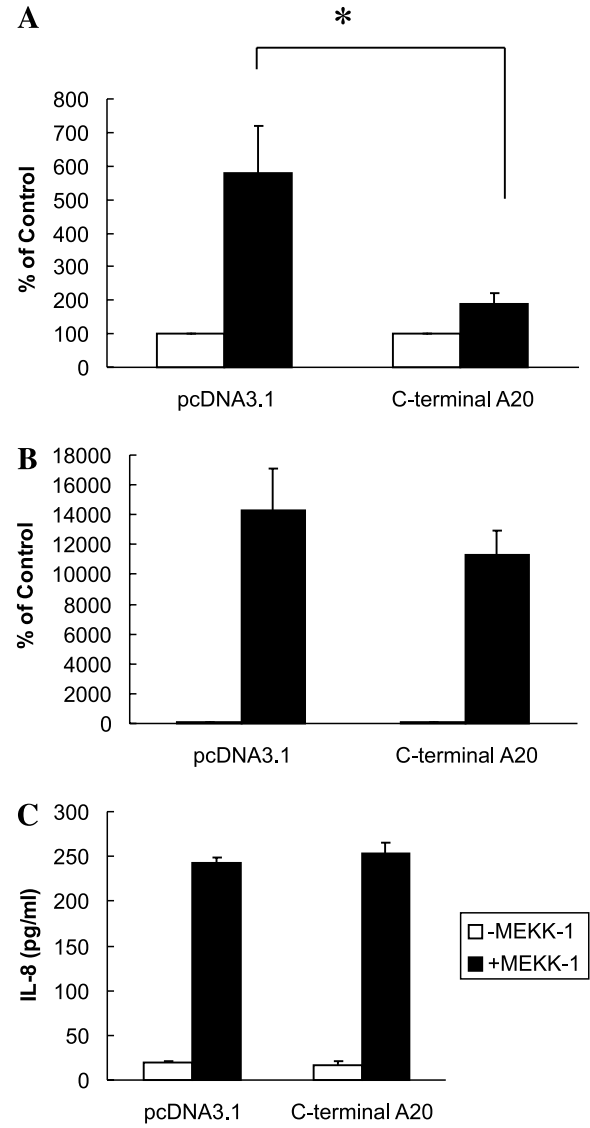

Fig. 4. Effects of C-terminal A20 on MEKK-1 signalling. (A,B) HEK293 cells were co-transfected with or without MEKK-1 (200 ng in (A); $50 \mathrm{ng}$ in (B)) in the presence or absence of C-terminal A20p3xFLAG-Myc-CMV (1 $\mu \mathrm{g})$ and (A) $\kappa$ B-luc $(0.5 \mu \mathrm{g})$ or (B) AP-1-luc $(0.5 \mu \mathrm{g})$. Cell extracts were subsequently measured for luciferase activity and protein content. Data are presented relative to cells transfected in the absence of MEKK-1. (C) HEK293 cells were co-transfected with or without MEKK-1 $(200 \mathrm{ng})$ in the presence or absence of C-terminal A20-p3xFLAG-Myc-CMV $(1 \mu \mathrm{g})$. Conditioned medium from cells was then assayed for IL- 8 by ELISA. Results represent means \pm SEM of three independent experiments $(*, p<0.05)$. 
C-terminal region of A20 on MEKK-1 activation of NF- $\kappa \mathrm{B}$ and AP-1 and induction of IL-8 (Fig. 4). The overexpression of C-terminal A20 was sufficient to strongly inhibit MEKK-1 activation of NF- $\kappa \mathrm{B}$ (Fig. 4A). However C-terminal A20 was ineffective in regulating the MEKK-1 activation of AP-1 (Fig. 4B) and also had no regulatory effects on MEKK-1 induction of IL-8 (Fig. 4C). Again the analogous effects of C-terminal A20 on both TLR-4 and MEKK-1 signalling provide support for A20 regulating TLR-4 activity at or downstream of MEKK-1.

\section{Discussion}

The tendency of A20-deficient mice to develop severe inflammation [16] coupled to their hyper-responsive nature to LPS suggests that A20 may act as an endogenous regulatory system in controlling the inflammatory response to gram-negative bacteria. This is further supported by the ability of LPS to increase the expression of A20 in endothelial cells [1]. The present study thus directly explored the ability of A20 to regulate the LPS signalling pathway with a view to increasing our mechanistic understanding of the anti-inflammatory effects of A20.

TLR-4 acts as the signalling receptor system for LPS and thus in order to examine the effects of A20 on LPS the influence of A20 on TLR-4 signalling was directly addressed. The study exploited CD4/TLR4, the constitutively active form of TLR-4 [17]. Full length A20 was

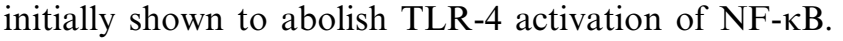
This mirrors the previously described inhibitory effects of A20 on IL- 1 and TNF activation of NF- $\kappa B$ [5]. Thus the present study demonstrates that A20 may be a universal inhibitor of the signalling pathways of key proinflammatory stimuli. However the inhibitory effects of A20 on LPS is not limited to blockade of NF- $\kappa \mathrm{B}$. This study also shows that A20 is an effective inhibitor of AP1 activation by TLR-4. Since NF- $\mathrm{BB}$ and AP-1 are key transcription factors in promoting the expression of a plethora of pro-inflammatory proteins the study subsequently examined the modulatory effects of A20 on IL-8 expression by TLR-4. IL-8 was chosen as a typical proinflammatory protein since it is prominently induced by LPS and plays a key role in promoting leukocyte recruitment into tissue during the inflammatory response [22]. Furthermore NF- $\mathrm{BB}$ and AP-1 are known to play key roles in regulating the expression of IL-8 [23] and it therefore allowed for a definition of the functional consequence of the inhibitory effects of A20 on the two transcription factors. A20 proved to be a powerful inhibitor of TLR-4 induction of IL-8. Such inhibitory effects of A20 on TLR-4 activation of NF- $\kappa$ B and AP-1 and induction of IL- 8 are likely to underlie the anti-inflammatory potential of A20 and form a plausible basis for explaining the hyper-responsive nature of A20-deficient mice to LPS.

It was previously known that the C-terminal zinc finger domain confers on A20 its ability to inhibit IL-1 activation of NF- $\mathrm{NB}$ [6-8]. This study demonstrated that the C-terminal regions of A20 is also sufficient to cause significant inhibition of TLR-4 activation of NF- $\kappa B$. This study for the first time also examined the ability of the C-terminal region of A20 to modulate activation of AP-1. The C-terminal region was shown to be ineffective in regulating TLR-4 activation of AP-1. Thus A20 demonstrates different structural requirements in its inhibition of NF- $\kappa \mathrm{B}$ and AP-1. The C-terminal region is sufficient to inhibit NF- $\kappa \mathrm{B}$ but ineffective in regulating AP-1. The N-terminal region appears to be required for manifesting the latter and this may be related to the ability of the N-terminal region to bind to signalling molecules in the TLR-4 pathway. It is interesting to note that the $\mathrm{N}$-terminal region of A20 has been shown to associate with TRAF-6 [7], a key signalling molecule in the TLR-4 pathway.

Interestingly C-terminal A20 failed to affect the ability of TLR-4 to induce IL-8. This may be due to its ineffectiveness in regulating AP-1. Nevertheless it is somewhat surprising considering that $\mathrm{C}$-terminal A20 acted as an effective inhibitor of NF- $\mathrm{KB}$. However whilst the inhibitory effects of C-terminal A20 on TLR-4 activation of NF- $\kappa B$ were strong, the inhibition was incomplete and thus the residual NF- $\kappa \mathrm{B}$ may be sufficient to co-operate with AP-1 in promoting IL-8 expression. Overall the studies show that the C-terminal region of A20 does not possess the same anti-inflammatory properties of the full length protein.

The possible target for A20 in its inhibition of TLR-4 signalling was next addressed. MEKK-1 was chosen as a lead candidate since it is known to regulate activation of both NF-kB and AP-1 [12-14] and a recent report had shown that A20 could inhibit MEKK-1 signalling at least with respect to NF- $\mathrm{BB}$ [15]. The present study confirms that A20 inhibits MEKK-1 activation of $\mathrm{NF}-\kappa \mathrm{B}$ and also for the first time shows that A20 inhibits MEKK-1 activation of AP-1. The similar effects of A20 on TLR-4 and MEKK-1 activation of NF- $\mathrm{KB}$ and AP-1 provides support for a model that A20 may regulate TLR-4 signalling at or downstream of MEKK1. However it is worth noting that the inhibition by A20 of MEKK-1 activation of AP-1 is partial unlike the total blockade of TLR-4 activation of AP-1. This may be due to the considerably more powerful stimulus provided by overexpression of MEKK-1 relative to CD4/TLR4. Furthermore the partial inhibition of MEKK-1 activation of AP-1 is also likely to explain the incomplete inhibitory effects of A20 on MEKK-1 induction of IL-8.

In order to further confirm that MEKK-1 is the likely target for A20 in the TLR-4 pathway the C-terminal 
zinc finger domain of A20 was also assessed for its regulatory effects on MEKK-1. C-terminal A20 regulated MEKK-1 signalling in a similar manner to its effects on TLR-4 signalling in that it inhibited MEKK-1 activation of NF- $\mathrm{KB}$ but was without effect on activation of AP-1 and induction of IL-8. The analogous effects of C-terminal A20 on TLR-4 and MEKK-1 signalling again supports MEKK-1 signalling as a likely target for A20 in the TLR-4 pathway.

The C-terminal A20 domain shares with full length A20 its ability to inhibit NF- $\kappa$ B but fails to replicate the inhibitory effects on AP-1. This suggests that the Nterminal domain of A20 is required for manifesting its inhibitory effects on AP-1 but is redundant for its regulation of NF- $\kappa$ B. This may be due to the ability of the $\mathrm{N}$-terminal and $\mathrm{C}$-terminal domains to interact with different signalling proteins. Thus the N-terminal domain is known to interact with TRAF proteins $[7,8]$ whereas the C-terminal region interacts with NEMO [11]. Since the latter is involved in promoting activation of NF- $\mathrm{KB}$ but not AP-1, the interaction of C-terminal A20 with NEMO may explain its inhibition of NF- $\kappa B$ but not AP-1. In contrast the interaction of the N-terminal domain of A20 with the TRAF proteins may be required for effecting inhibition of AP-1.

In summary this study demonstrates for the first time that the anti-inflammatory and regulatory effects of A20 on TLR-4 may be mediated by regulating MEKK-1 activation of NF- $\kappa$ B and AP-1. Furthermore it also defines different structural requirements of A20 for inhibiting the two transcription factors. This is of value in increasing our mechanistic understanding of the anti-inflammatory effects of A20. A full appreciation of the mechanism(s) may help in the therapeutic exploitation of A20 as a novel anti-inflammatory strategy.

\section{Acknowledgments}

This work was supported by the European Biotechnology 5th Framework Programme (Contract No. QLG1-CT-1999-00549), Health Research Board of Ireland and Enterprise Ireland.

\section{References}

[1] V.M. Dixit, S. Green, V. Sarma, L.B. Holzman, F.W. Wolf, K. O'Rourke, P.A. Ward, E.V. Prochownik, R.M. Marks, Tumor necrosis factor- $\alpha$ induction of novel gene products in human endothelial cells including a macrophage-specific chemotaxin, J. Biol. Chem. 265 (1990) 2973-2978.

[2] V. Sarma, Z. Lin, L. Clark, B.M. Rust, M. Tewari, R.J. Noelle, V.M. Dixit, Activation of the B-cell surface receptor CD40 induces A20, a novel zinc finger protein that inhibits apoptosis, J. Biol. Chem. 270 (1995) 12343-12346.

[3] C.D. Laherty, H.M. Hu, A.W. Opipari, F. Wang, V.M. Dixit, The Epstein-Barr virus LMP1 gene product induces A20 zinc finger protein expression by activating nuclear factor $\kappa \mathrm{B}, \mathrm{J}$. Biol. Chem. 267 (1992) 24157-24160.

[4] A. Krikos, C.D. Laherty, V.M. Dixit, Transcriptional activation of the tumor necrosis factor $\alpha$-inducible zinc finger protein, A20, is mediated by $\kappa$ B elements, J. Biol. Chem. 267 (1992) 17971-17976.

[5] M. Jaattela, H. Mouritzen, F. Elling, L. Bastholm, A20 zinc finger protein inhibits TNF and IL-1 signaling, J. Immunol. 156 (1996) 1166-1173.

[6] M. Klinkenberg, S. Van Huffel, K. Heyninck, R. Beyaert, Functional redundancy of the zinc fingers of A20 for inhibition of NF- $\mathrm{\kappa B}$ activation and protein-protein interactions, FEBS Lett. 498 (2001) 93-97.

[7] K. Heyninck, R. Beyaert, The cytokine-inducible zinc finger protein A20 inhibits IL-1-induced NF- $\kappa \mathrm{B}$ activation at the level of TRAF6, FEBS Lett. 442 (1999) 147-150.

[8] H.Y. Song, M. Rothe, D.V. Goeddel, The tumor necrosis factorinducible zinc finger protein A20 interacts with TRAF1/TRAF2 and inhibits NF-kB activation, Proc. Natl. Acad. Sci. USA 93 (1996) 6721-6725.

[9] K. Heyninck, D. De Valck, W. Vanden Berghe, W. Van Criekinge, R. Contreras, W. Fiers, G. Haegeman, R. Beyaert, The zinc finger protein A20 inhibits TNF-induced NF-кBdependent gene expression by interfering with an RIP- or TRAF2-mediated transactivation signal and directly binds to a novel NF-кB-inhibiting protein ABIN, J. Cell. Biol. 145 (1999) 1471-1482.

[10] S. Van Huffel, F. Delaei, K. Heyninck, D. De Valck, R. Beyaert, Identification of a novel A20-binding inhibitor of nuclear factor$\kappa \mathrm{B}$ activation termed ABIN-2, J. Biol. Chem. 276 (2001) 3021630223.

[11] S.Q. Zhang, A. Kovalenko, G. Cantarella, D. Wallach, Recruitment of the IKK signalosome to the p55 TNF receptor: RIP and A20 bind to NEMO (IKK $\gamma$ ) upon receptor stimulation, Immunity 12 (2000) 301-311.

[12] C.H. Regnier, H.Y. Song, X. Gao, D.V. Goeddel, Z. Cao, M. Rothe, Identification and characterization of an IкB kinase, Cell 90 (1997) 373-383.

[13] N.L. Malinin, M.P. Boldin, A.V. Kovalenko, D. Wallach, MAP3K-related kinase involved in NF- $\mathrm{KB}$ induction by TNF, CD95 and IL-1, Nature 385 (1997) 540-544.

[14] A.J. Whitmarsh, R.J. Davis, Transcription factor AP-1 regulation by mitogen-activated protein kinase signal transduction pathways, J. Mol. Med. 74 (1996) 589-607.

[15] F.S. Zetoune, A.R. Murthy, Z. Shao, T. Hlaing, M.G. Zeidler, Y. $\mathrm{Li}, \mathrm{C}$. Vincenz, A20 inhibits NF- $\mathrm{kB}$ activation downstream of multiple Map3 kinases and interacts with the IкB signalosome, Cytokine 15 (2001) 282-298.

[16] E.G. Lee, D.L. Boone, S. Chai, S.L. Libby, M. Chien, J.P. Lodolce, A. Ma, Failure to regulate TNF-induced NF- $\kappa B$ and cell death responses in A20-deficient mice, Science 289 (2000) 2350-2354.

[17] R. Medzhitov, P. Preston-Hurlburt, C.A. Janeway Jr., A human homologue of the Drosophila Toll protein signals activation of adaptive immunity, Nature 388 (1997) 394-397.

[18] A. Poltorak, X. He, I. Smirnova, M.Y. Liu, C.V. Huffel, X. Du, D. Birdwell, E. Alejos, M. Silva, C. Galanos, M. Freudenberg, P. Ricciardi-Castagnoli, B. Layton, B. Beutler, Defective LPS signaling in $\mathrm{C} 3 \mathrm{H} / \mathrm{HeJ}$ and $\mathrm{C} 57 \mathrm{BL} / 10 \mathrm{ScCr}$ mice: mutations in Tlr4 gene, Science 282 (1998) 2085-2088.

[19] J.C. Chow, D.W. Young, D.T. Golenbock, W.J. Christ, F. Gusovsky, Toll-like receptor-4 mediates lipopolysaccharide-induced signal transduction, J. Biol. Chem. 274 (1999) 1068910692.

[20] M. Muzio, G. Natoli, S. Saccani, M. Levrero, A. Mantovani, The human Toll signaling pathway: divergence of nuclear factor $\kappa \mathrm{B}$ and JNK/SAPK activation upstream of tumor necrosis factor receptor-associated factor 6 (TRAF6), J. Exp. Med. 187 (1998) 2097-2101. 
[21] M.M. Bradford, A rapid and sensitive method for the quantitation of microgram quantities of protein utilizing the principle of protein-dye binding, Anal. Biochem. 72 (1976) 248-254.

[22] Z.M. Wang, C. Liu, R. Dziarski, Chemokines are the main proinflammatory mediators in human monocytes activated by
Staphylococcus aureus, peptidoglycan and endotoxin, J. Biol. Chem. 275 (2000) 20260-20267.

[23] N. Mukaida, S. Okamoto, Y. Ishikawa, K. Matsushima, Molecular mechanism of interleukin-8 gene expression, J. Leukoc. Biol. 56 (1994) 554-558. 